Voix et Images

\title{
Alexandre Chenevert et Kamouraska : une lecture australienne
}

\section{Grahame C. Jones}

Volume 7, numéro 2, hiver 1982

Michel Tremblay

URI : https://id.erudit.org/iderudit/200323ar

DOI : https://doi.org/10.7202/200323ar

Aller au sommaire du numéro

Éditeur(s)

Les Presses de l'Université du Québec

ISSN

0318-9201 (imprimé)

1705-933X (numérique)

Découvrir la revue

Citer cet article

Jones, G. C. (1982). Alexandre Chenevert et Kamouraska : une lecture australienne. Voix et Images, 7(2), 329-341. https://doi.org/10.7202/200323ar d'utilisation que vous pouvez consulter en ligne.

https://apropos.erudit.org/fr/usagers/politique-dutilisation/ 


\title{
Alexandre Chenevert et Kamouraska : une lecture australienne
}

\author{
par Grahame C. Jones
}

Une phrase de Gabrielle Roy a retenu mon attention. Elle est tirée d'Alexandre Chenevert:

Mais quelle chance l'homme a-t-il de rencontrer parmi les millions d'habitants du globe son âme sœur qui est peut-être un Chinois ou un Australien ! 1

Évidemment, je ne peux pas parler pour les Chinois. Je peux cependant affirmer qu'Alexandre Chenevert a trouvé des lecteurs compréhensifs parmi les Australiens. Si la littérature québécoise est enseignée actuellement à l'Université de New England, si elle a provoqué tant d'intérêt chez nos étudiants, cela est en partie dû au grand succès, sur le plan pédagogique, de deux romans : Alexandre Chenevert de Gabrielle Roy et Kamouraska d'Anne Hébert ${ }^{2}$. Ce que j'entends faire à présent, c'est d'examiner et de comparer ces deux ouvrages dans le but de savoir s'ils ont des éléments communs qui nous permettraient de comprendre leur attrait universel.

Un tel projet pourrait sembler assez surprenant, car la remarquable Elisabeth d'Aulnières de Kamouraska ne parait pas avoir, à première vue, beaucoup en commun avec l'humble Alexandre Chenevert. Quels rapports peut-il y avoir entre la célèbre veuve d'Antoine Tassy, - personnage si notoire qu'elle n'ose même pas sortir dans les rues de peur d'être reconnue comme la vedette d'un procès sensationnel ( On se retourne sur mon passage. C'est cela ma vraie vie. Sentir le monde se diviser en deux haies pour me voir passer" ${ }^{3}$, - et ce petit employé de banque qu'est Chenevert, être si insignifiant qu'il passerait sûrement inaperçu, ou peut-être pas inaperçu, car il est impossible de se promener dans les rues à certaines heures sans le voir partout, tant il ressemble à la multitude humaine ( $\mathrm{Le}$ matin, à heure fixe, il descendait de mille escaliers à la fois, courant de tous les points de la ville vers des trams archicombles. Il s'y entassait à cent, à mille exemplaires. De tram en tram, de rue en rue, on le voyait debout dans les véhicules, les mains passées dans une courroie de cuir, les bras étirés dans une curieuse pose de supplicié») ${ }^{4}$. Certes, Elisabeth et Alexandre se trouvent, à bien des égards, aux deux extrêmes de l'échelle 
humaine. Néanmoins, j'espère pouvoir montrer, sans minimiser cette différence, que ces deux personnages sont plus semblables qu'on ne pourrait s'y attendre, de sorte qu'il serait légitime de se demander si nous avons affaire ici à des éléments d'un imaginaire québécois, ce qui expliquerait dans quelque mesure l'attrait commun de ces deux œuvres apparemment si disparates.

Si différents que soient leurs origines et leurs milieux sociaux, on trouve Alexandre et Elisabeth, au début de leurs romans respectifs, dans la même situation morale. Chacun attend l'aube, incapable de dormir, torturé par une incessante activité cérébrale, se demandant quel est le sens de la vie qu'il mène. «ll avait, dans sa vie, perdu quantité de choses, et presque toujours les meilleures : la jeunesse d'abord, ensuite la santé; et maintenant le sommeil", nous dit Gabrielle Roy de son protagoniste ${ }^{5}$. Quant à Elisabeth d'Aulnières, elle est déchirée entre le besoin de dormir («Cette fièvre de l'insomnie si tu savais, Jérôme mon mari, comme je la partage avec toi ${ }^{6}$, et la peur de s'endormir et de faire face ainsi aux vérités effrayantes de ses rêves ("Tout, pour ne pas succomber au sommeil", se promet-elle ailleurs, $)^{7}$. Ces heures d'insomnie mettent en relief la profonde solitude des deux personnages. Alexandre se sent de plus en plus séparé d'une humanité qui est représentée pour lui par les longues files de gens qu'il voit partout - et surtout devant son guichet à la banque.

II ferma les yeux passionnément intéressé par ce problème d'identité individuelle que poserait le Jugement dernier (...) toutes les files de la terre, les patientes files de tous les temps s'allongeaient les unes au bout des autres, se repliaient sur elles-mêmes comme des routes en montagne $(\ldots)^{8}$.

II croit habiter un monde où aucun contact valable n'est plus possible entre les gens. Sa femme, sa fille, son meilleur ami, sont tous des solitudes impénétrables pour lui : ils ne veulent même pas écouter les idées sérieuses qui traversent sans cesse son esprit.

La situation d'Elisabeth n'est pas moins pénible. Elle a perdu le seul être fait pour la comprendre. Sa famille nombreuse lui fait sentir de façon désespérée son profond isolement. C'est en vain qu'elle s'entoure des siens comme pour se protéger contre les forces qui menacent de la détruire: "Réveiller tous les enfants. S'en faire un rempart. Les lâcher dans la maison, les mettre aux fenêtres, les poster à la porte de la rue " ${ }^{9}$; c'est en vain qu'elle s'imagine, à l'instar de la reine Victoria, entourée de ses enfants, sûre de son rôle social ( "Je ressemble à la reine d'Angleterre" ${ }^{10}$. Aucun subterfuge ne saurait cacher sa différence fondamentale : elle est déplacée dans ce décor bourgeois. Elle est rejetée par les autres, elle est condamnée à la solitude. Une image qu'elle emploie elle-même à la fin du roman éclaire sa situation. Elle s'imagine dans l'auberge où le docteur Nelson s'est rendu après son crime, mais elle se trouve incapable de l'aider, de le réconforter. "Me voici emmurée dans ma propre solitude", dit-elle "11. Les vraies barricades qui la cernent, ne sont pas les remparts qu'elle voulait construire 
avec ses propres enfants, mais les murs de la solitude. Elle touche ici à un principe essentiel de la vie humaine, principe que découvre aussi Alexandre Chenevert : "Trop d'espace au dehors, pas assez à l'intérieur; la curieuse loi de la vie solitaire le décontenançait" ${ }^{12}$.

Cependant, cet étroit espace intérieur de notre solitude ne reste pas longtemps inhabité. Il devient le lieu d'une rencontre de l'individu avec lui-même. Au plus profond de lui, Alexandre entretient une riche vie spirituelle imperceptible aux autres. Son existence est divisée en deux: l'ingrate routine quotidienne, qui lui devient de plus en plus étrangère, et la vraie vie, la vie authentique, qu'est sa méditation intérieure.

Donc une vie pour les nécessités : s'habiller, payer les meubles, le loyer, le chauffage et l'électricite; puis une autre vie, celle-ci toute de méditation comme celle de Gandhi dans son pagne blanc ${ }^{13}$.

Sa vie intérieure l'introduit dans un domaine de souffrance où il peut assumer les peines de toute l'humanité. Elle ajoute à son existence une dimension métaphysique : elle lui permet d'accéder à un domaine privilégié où la vie est vécue avec une intensité étonnante. Cela explique la frustration qui l'envahit chaque fois que les petits soucis de son emploi empiètent sur ses expériences méditatives. Un jour qu'il compte sa monnaie, il subit une révélation mystique d'une clarté fulgurante : “Et d'ici même, comme il attaquait sa trentième dizaine, Alexandre aperçut l'éternité »14. La simplicité de cette phrase, avec son vocabulaire humblement quotidien, est tout à fait saisissante. Pour trouver d'autres exemples où une profonde illumination religieuse est exprimée avec une candeur aussi modeste il faudrait les chercher chez les poètes anglais de l'école métaphysique, chez Henry Vaughan, par exemple, qui commence un de ses poèmes en disant sans ambages: "I saw eternity the other night". Alexandre n'a pourtant pas le temps d'approfondir cette révélation; et la romancière nous transmet la déception de son personnage : «ll ne pouvait tout de même pas être dans le même instant et à ses cents et à son âme" ${ }^{15}$. Eugène lonesco a bien expliqué, dans son théâtre, comment l'homme moderne risque d'être anéanti par son rôle social : «(...) il est aboli par sa fonction, il n'est plus que sa fonction aliénante, il n'est plus". Ce qui compte, évidemment, ce n'est pas la "fonction aliénante" que nous devons remplir (fonction que Gabrielle Roy désigne, dans le cas de son personnage, en l'appelant de manière impersonnelle $A$. Chenevert ${ }^{16}$ puisque ce titre brille "en lettres sombres" sur la plaque posée sur son comptoir), mais l'être qui se cache à l'intérieur de nous. Chez Alexandre cet être secret rappelle Gandhi, le grand Indien humaniste auquel Alexandre ressemble physiquement et à qui il voudrait ressembler sur le plan moral aussi.

Elisabeth connaît le même conflit entre la "fonction aliénante" imposée par la société et l'être qu'elle entretient au fond d'elle. Le dégoût qu'elle éprouve pour son rôle social est patent. On voit bien la répugnance avec laquelle elle évoque cette Mme Rolland qu'elle est devenue mais dont elle parle de préférence à la troisième personne, n'employant jamais de 
prénom, comme s'il s'agissait d'une autre qu'il fallait traiter avec politesse et tenir à distance. Elle dit avec indignation comme si elle était la victime d'un malentendu sur son identité : “On va jusqu'à prétendre que je suis Mme Rolland, épouse de Jérôme Rolland, notaire de cette ville..." "17. Mais sa situation est plus compliquée que celle d'Alexandre. Si elle est portée vers un passé qui semble contenir sa véritable identité, elle recule parfois avec horreur devant ce même passé parce qu'il renferme tant d'événements tragiques. Par moments elle cherche avec soulagement à s'accrocher à l'existence banale mais bien déterminée de Mme Rolland: "Je n'ai rien à faire rue Augusta, à Sorel. Je vous le jure. Je suis Mme Rolland, Mme Jérôme Rolland" ${ }^{18}$. Ce qu'elle cherche dans son rôle bourgeois, c'est une essence (au sens sartrien du terme), une garantie de son innocence démontrée par dix-huit ans de fidélité conjugale: «Je suis innocente!» proteste-t-elle à plusieurs reprises - mais sans jamais réussir à se convaincre.

La vie intérieure d'Elisabeth constitue un va-et-vient perpétuel entre un présent qui la repousse et un passé qui l'attire mais qui l'épouvante. Elle ne sait pas où se réfugier. Elle occupe une sorte de no man's land, perdue entre ces deux plans temporels. "Ma vie est ailleurs. Toute retirée dans un lieu vague. Une espèce de campagne désaffectée où l'effroi fait des ombres chinoises"19. Sa vie n'est pas simplement divisée en deux, comme celle d'Alexandre; elle est brisée en éclats dont chacun représente une facette de son identité.

C'est peu d'avoir une double vie, madame Rolland. Le plus difficile serait d'avoir quatre ou cinq existences secrètes, à l'insu de tous ${ }^{20}$.

Le catalogue des noms qui sont employés à son égard fait ressortir la crise d'identité qujelle traverse : elle est la Petite, Elisabeth d'Aulnières, Elisabeth Tassy, Mme Jérôme (jamais Elisabeth) Rolland. Les fluctuations de l'emploi des pronoms reflètent aussi l'incertitude de sa vie personnelle. Souvent sa vie est racontée à la troisième personne. Technique dont l'intention est de montrer un certain détachement, sinon une certaine répulsion de la part d'Elisabeth, un refus de s'identifier avec la personne qu'elle est. C'est ce qui arrive, par exemple, quand elle s'appelle Mme Rolland ou qu'elle se sert des termes le marié l la mariée pour raconter son long voyage vers Kamouraska avec son premier mari. Pourtant, il arrive que les pronoms changent irrésistiblement pour la situer à l'intérieur du personnage. Un excellent exemple de cette tendance se trouve dans l'extrait suivant où Elisabeth décrit à la troisième personne sa première communion, mais où elle finit par s'identifier au jeune être qu'elle voulait traiter avec un détachement ironique.

Dans cet espace réduit, dans cet air gris qui se raréfie, surgit une communiante. Tout de blanc vêtue, de la tête aux pieds. Son long voile pend jusqu'à terre. Sur sa tête une couronne de roses blanches. Je ne puis faire un mouvement. Dans sa main lourde, dans mon bras pétrifié, doucement meurt l'esquisse vaine d'un signe de croix. Un 
enfant qui est moi me regarde, bien en face, et me sourit gravement. M'oblige à écouter la voix légère et solennelle que je croyais perdue ${ }^{21}$.

Ici, et dans d'autres passages similaires, l'alternance des pronoms traduit la tension qui existe chez un être résolu à s'échapper de son passé mais contraint d'assumer l'identité qui était la sienne.

Encore plus intéressant, en ce qui concerne le thème de l'aliénation dans Kamouraska est l'emploi de la narration à la deuxième personne. Cette technique a beaucoup intéressé Michel Butor qui en décrit les caractéristiques de la façon suivante:

C'est ici qu'intervient l'emploi de la seconde personne, que l'on peut caractériser ainsi dans le roman : celui à qui l'on raconte sa propre histoire.

C'est parce qu'il y a quelqu'un à qui l'on raconte sa propre histoire, quelque chose de lui qu'il ne connaît pas, ou du moins pas encore au niveau du langage qu'il peut y avoir un récit à la seconde personne, qui sera par conséquent toujours un récit « didactique ${ }^{22}$.

Ce ton «didactique» est apparent dans les passages où Elisabeth, dans son imagination, s'adresse à ceux qui sont moins intelligents, moins sensibles, qu'elle. Notez le ton de condescendance avec lequel elle déplore le peu d'expérience de ses tantes: "Pauvres saintes femmes de la rue Augusta. Vous ne comprenez rien à rien", etc. ${ }^{23}$. Le procédé révèle son plein pouvoir, cependant, lorsqu'Elisabeth recourt au pronom à la deuxième personne pour raconter les fluctuations de sa propre vie intérieure. En voici un exemple :

Bonjour madame Rolland. Bonsoir madame Rolland. Comment va monsieur Rolland? Et les enfants? Une fameuse portée, mais tous en bonne santé. Dieu merci. À quoi pensez-vous donc, madame Rolland, que votre œil s'assombrit? Que votre front se plisse? Irréprochable. Vous êtes irréprochable. Mais vous n'êtes qu'une absente, madame Rolland. Inutile de nier. Votre mari se meurt dans une des chambres du premier et vous feignez de dormir, étendue sur le lit de l'institutrice de vos enfants. Vous entendez des voix, madame Rolland. Vous jouez à entendre des voix. Vous avez des hallucinations. Avez-vous donc tant besoin de distractions qu'il vous faut aller chercher au plus creux des ténèbres, les fantômes de votre jeunesse ? ${ }^{24}$

L'usage de vous dans ce passage introduit une note autoritaire. Mme Rolland est harcelée de questions comme une criminelle à un interrogatoire. Cependant, ces questions ne sollicitent pas de réponse. Ce sont des affirmations déguisées en questions, des accusations qui mettent en cause la sincérité de celle à qui elles sont adressées. Benveniste nous dit que le pronom je implique l'existence du $t u$ : il s'ensuit que le $t u$ suppose l'existence du je. Qui, donc, est le je auteur de ces questions, le je absent, puisque le pronom à la première personne ne paraît pas dans ce paragraphe? N'oublions pas que Kamouraska est une aventure passionnée racontée telle qu'elle est revécue dans la conscience d'Elisabeth. Cela étant, l'imagina- 
tion qui conçoit ces questions est celle du protagoniste du roman. La situation est donc différente de celle de la Modification de Butor où le vous est prononcé par un narrateur omniscient qui fait la leçon à un personnage ignorant sur lui-même. Dans Kamouraska celle qui interroge et celle qui est interrogée sont la même personne. Cette division en éléments antagonistes d'une même sensibilité renforce le sentiment d'aliénation.

A d'autres moments Elisabeth emploie le tu pour faire ressortir son drame intérieur. Le tutoiement introduit, bien entendu, une note de familiarité. Il peut servir à montrer du mépris pour un inférieur ou à indiquer la supériorité qu'on veut afficher vis-à-vis de quelqu'un de plus jeune, de plus inexpérimenté. Le passage suivant révèle la condescendance que témoigne Elisabeth à l'égard de la petite niaise qu'elle a été lors de son premier mariage. Cet extrait est d'ailleurs fort intéressant parce que le changement des pronoms personnels (où le je remplace le tu comme sujet des deux dernières phrases) nous fait comprendre l'identité de celle qui parle et de celle dont elle parle et implique celle-là dans la vie intérieure de celle-ci.

Ton voile de mariée. Ta couronne de fleurs d'oranger. Ta robe à traîne. Le gâteau de noces, à trois étages, nappé de sucre et de crème fouettée. Les invités se mouchent derrière toi. Tout le bourg de Sorel attend pour te voir passer, au bras de ton jeune époux. Mon Dieu, je me damne! Je suis mariée à un homme que je n'aime pas ${ }^{25}$.

Dans les deux romans il y a donc un conflit entre la réalité quotidienne et la vie intérieure. Chez Elisabeth cette existence intime correspond à la vraie vie, à celle où elle a été elle-même avant de devenir Mme Rolland.

J'habite ailleurs. Un lieu précis. Un temps révolu. Aucun prestige de la mémoire ne pourrait réussir cela. Il s'agit de la possession de ma vie réelle. De ma fuite parfaite de la rue du Parloir. ${ }^{26}$

Son aventure ne se passe pas dans quelque domaine irréel : elle est ce qui a vraiment eu lieu. Pour Alexandre Chenevert, au contraire, l'aventure est reléguée à l'univers de l'impossible. Elle est lointaine, comme les îles de corail dans les romans d'aventure. Néanmoins il vient un moment de sa vie où les rêves d'Alexandre semblent réalisables. II s'agit des vacances qu'il passe au bord du lac Vert. Là il se trouve en communion avec la Nature: il y découvre une paix qu'il croyait impossible. II comprend le message des arbres : “Les arbres s'inclinaient, ils disaient à Alexandre qu'ils vivaient un temps, mouraient, étaient remplacés, et que tout était bien ainsi»" ${ }^{27}$. Son bonheur rappelle celui auquel pense Albert Camus quand, réfléchissant sur l'Absurde de notre condition, il affirme dans le Mythe de Sisyphe : "Si j'étais arbre parmi les arbres, chat parmi les animaux, ce monde aurait un sens, ou plutôt ce problème n'en aurait point (...)",28. Pour Alexandre l'absurde routine cyclique est remplacée par le cycle harmonieux de la Nature. Sa vie en est transformée, de sorte que la solitude devient pour lui non plus ce vide angoissant de ses nuits d'insomnie, mais une profonde expérience quasi religieuse. II serait légitime de croire qu'Alexandre est arrivé au terme de son itinéraire spirituel. Mais le roman ne s'arrête pas là. Pas plus que le 
Candide de Voltaire à Eldorado, Alexandre ne peut accepter le bonheur s'il lui faut se détourner du monde humain. Sa félicité parfaite ne dure qu'une journée : comme Candide, il s'ennuie au Paradis. 'Il s'aperçoit de la vraie nature du bonheur qu'il partage avec les Le Gardeur, ses voisins :

De quoi était fait ce bonheur, au fond, sinon de suffisance? Les Le Gardeur étaient heureux parce qu'ils n'avaient besoin de personne au monde. «Parce que je manque ni de vivres, ni de feu, parce que je ne suis pas malade, je considere Dieu mon bienfaiteur. " Au fond, de quoi l'homme heureux rendait-il grâce,sinon de l'inégalité sur terre ? ${ }^{29}$

A l'instar de Camus il avait rêvé de partager le bonheur des arbres: «(...) il s'identifia avec une secrète entente du cœur aux éléments irresponsables et dociles de la création ${ }^{30}$; à présent les arbres auxquels il s'identifie sont ceux qui chantent non le bonheur, mais le malheur: "Entre tous les arbres, Alexandre préférait maintenant ceux-ci qui ne s'arrêtaient pas de se plaindre " ${ }^{31}$. De sa solitude il rêve de composer une lettre sur l'interdépendance de tous les êtres humains. Cependant, il ne trouve pas les mots pour s'exprimer. II a rejeté la solitude comme solution factice et égoïste, mais il se trouve incapable de communiquer l'essentiel de ce qu'il a à dire.

Nous touchons à présent au problème de l'Autre. Cette question a préoccupé bien des penseurs, y compris Benjamin Constant qui l'a résumée en une phrase instructive. "Les autres sont les autres, on ne fera jamais qu'ils soient 'soi' »32. Alexandre et Elisabeth cherchent à résoudre le problème de la façon la plus radicale: par l'Amour. À l'incompréhension qui distingue à l'ordinaire la relation entre les êtres ils veulent substituer un accord parfait. Chez Elisabeth l'amour prend la forme d'une plongée étroite et profonde dans sa vie passée et il se concentre sur une seule relation. Cette relation idéale trouve sa contrepartie ironique dans la description de la vie conjugale d'Elisabeth avec son deuxième mari :

Épouse parfaite de Jérôme Rolland, un petit bonhomme doux qui réclame son dû presque tous les soirs, avant de s'endormir, jusqu'à ce qu'il en devienne cardiaque. Mon devoir conjugal sans manquer. Règles ou pas. Enceinte ou pas. Nourrice ou pas. Parfois même le plaisir amer. L'humiliation de ce plaisir volé à l'amour. Pourquoi faire tant de simagrées. Je n'ai été qu'un ventre fidèle, une matrice à faire des enfants. ${ }^{33}$

Telle est sa vie actuelle. Mais dans sa fantaisie elle revit l'amour qu'elle a connu autrefois et qui reste l'expérience cruciale de sa vie : "L'amour meurtrier. L'amour infâme. L'amour funeste. Amour. Amour. Unique vie de ce monde. La folie de l'amour ' ${ }^{34}$. Chez Alexandre Chenevert la même impulsion prend une dimension moins temporelle que spatiale. Son désir de l'amour s'étend pour embrasser toute l'humanité : «ll ne faisait pas de doute qu'en ce moment il aimait tous ses compagnons de la terre" ${ }^{35}$. Cet amour-charité participe de l'universel. Quelle est la clef de l'empathie d'Alexandre? II a découvert, dans sa vie spirituelle, les profondes implications philosophiques et morales d'une technique foncièrement littéraire: 
l'emploi du point de vue narratif. Pour éprouver de la sympathie pour un autre il suffit de nous mettre à sa place, de voir le monde comme il le fait : «Si j'étais le Président Truman, proposa Alexandre, qu'est-ce que je ferais? Et il eut pitié du président Truman» ${ }^{36}$. Les conséquences d'un pareil exercice mental sont souvent déroutantes et donc inacceptables. Par exemple, monsieur Fontaine, le directeur de la banque d'Alexandre, est bouleversé lorsque, d'habitude homme de très peu d'imagination, il réussit de façon inattendue à voir les choses à travers la conscience de son employé :

Il lui arriva de convenir que le monde, aux yeux d'un caissier, pouvait bien ne pas offrir de puissant encouragement à la joie; il se prit à l'examiner avec un regard de caissier, si possible. Mais aussitôt il fut indigné de ce qu'il apercevait: le monde entier à changer à cause d'un seul petit homme. ${ }^{37}$

Que découvre-t-on en adoptant une série suffisamment variée de points de vue différents? Que le monde est à refaire. Fontaine, bon bourgeois profondément conservateur, recule devant les conséquences de cet acte d'imagination : Alexandre, au contraire, les accepte sans résenve. J'ai cité plus haut une phrase dans laquelle Benjamin Constant affirme que le problème de l'Autre existe parce qu'on ne peut pas faire qu'il s'identifie à nous. Pour Alexandre le problème devient soluble dès qu'on adopte le processus contraire : c'est à nous de nous identifier à lui.

La question de l'Autre ne se borne pourtant pas à notre observation à nous de ceux qui nous entourent. Elle est d'autant plus complexe que nous aussi, nous sommes des Autres aux yeux d'autrui. Selon une maxime célèbre de Jean-Paul Sartre, le service le plus indésirable que nous rend l'Autre, c'est de nous fournir un dehors. Pour éclairer les implications de cette situation il serait utile d'emprunter, à un article que Sartre a consacré aux romans de Mauriac ${ }^{38}$, les termes il-sujet et il-objet et de les appliquer dans un domaine philosophique plutôt que littéraire. En général l'Autre est pour nous un il-objet. II est opaque, parce que vu du dehors. On le traite comme s'il n'avait pas de vie intérieure, alors que la vie intérieure est le centre de son existence. Les expériences qu'entreprend Alexandre Chenevert quand il essaie de voir le monde d'un point de vue différent, correspondent donc à une tentative de sa part de voir l'Autre non comme un il-objet, mais comme un il-sujet. Le grand malheur d'Alexandre, c'est que les Autres ne veulent pas faire le même effort pour lui. $A$ leurs yeux il est un objet, un numéro ( «le petit monsieur du 8846 " 39 . Il est tellement déshumanisé par cette manière de voir qu'il se rend compte qu'il est réduit dans l'imagination d'autrui à la fonction visible qu'il remplit dans la société.

(...) il [un des clients de la banque] regardait le caissier avec insistance, sans intérêt toutefois, il le regardait comme si celui-ci eût été une machine. Alexandre croisa ce regard qui le voyait comme une machine. ${ }^{40}$

Alexandre est le plus généreux des hommes, mais il est condamné à être un misanthrope désagréable par ce regard d'autrui qui ne pénètre jamais jusqu'à sa vie intérieure. 
Dans le cas d'Elisabeth le conflit entre le sujet que nous sommes pour nous-mêmes et l'objet que nous sommes pour autrui donne lieu à une série de conséquences très contradictoires. Elle sait, tout aussi bien qu'Alexandre, qu'aux yeux des Autres nous sommes toujours réduits à notre rôle social. Cependant, plutôt que de réagir avec aversion contre une telle injustice, elle tâche souvent de l'exploiter, de la tourner à son avantage. Elle souhaite d'être confondue avec sa fonction sociale ("Il fallait me refaire une innocence à chaque séance, comme une beauté entre deux bals, une virginité entre deux hommes " ${ }^{4}$. Étre un objet, c'est quand même éviter les souffrances qui proviennent de notre monde subjectif. Mais le regard qu'elle sent braqué sur elle, est un regard accusateur. Les autres sont pour elle des témoins, des témoins à charge. Comme le Meursault de l'Étranger, elle est incapable de savoir que les autres la regardent, sans conclure que c'est pour la juger et la condamner. Ce regard la poursuit partout, même dans la solitude :

- C'est elle qui a tué son mari! Cette femme est une criminelle. Voyez comme elle traîne dans la rue, en pleine nuit. ${ }^{42}$

Sa plus grande crainte, c'est que tous ces témoins hostiles viennent lui faire subir les conséquences de ses actions:

Tout ce bavardage des témoins autour de mon lit (...). Ils prennent en secret des mesures exemplaires pour me sortir de mon lit. M'arracher à jamais de ma maison de la rue du Parloir. ${ }^{43}$

Evidemment, cette condamnation aurait été inefficace, si Elisabeth avait refusé d'en tenir compte. Braver le scandale, provoquer le jugement, mais le refuser et vivre en conséquence, c'est ce qu'elle aurait pu faire, ce qu'elle a voulu faire lorsqu'elle a lié son destin à celui du docteur Nelson. Voilà pourquoi, une nuit, elle se tient debout, toute nue, devant la fenêtre à côté de son amant dans l'espoir d'être vue par les passants, pourquoi elle proclame sa révolte en s'écriant:

II s'agit maintenant de nous compromettre à jamais. De provoquer le scandale. D'accepter sans retour qu'on nous accuse et nous montre du doigt. Tous deux liés ensemble dans une seule nécessité. Ayant rompu avec le monde. L'absolu de l'amour et de la mort. La justice rétablie. ${ }^{44}$

Cependant, le roman d'amour qu'elle revit dans son imagination est un roman inachevé. Elle ne pourra jamais le compléter en s'enfuyant pour rejoindre son amant. Tout ce qu'elle peut faire, c'est de le revivre incessamment dans ses rêves et de se résigner à souffrir à jamais les conséquences de son hypocrite vie double.

Il suffit de voir combien le monde est transformé dans la conscience de ces deux protagonistes pour comprendre à quel point l'univers fictif présenté dans chacun des romans est la création de l'imagination qui le sous-tend. Alexandre est comme un Atlas, ainsi que le comprend son médecin : 
On ne peut pas vivre indéfiniment tendu comme vous l'êtes. Vous pensez trop. Vous raisonnez trop. Que diable, fit-il, vous portez le monde sur vos épaules. 45

Alexandre agit comme si toute la création dépendait de lui :

II marchait, soucieux et, comme s'il eût été Dieu, s'inquiétant de sa souffrance en des vies issues de lui et jusqu'en des générations à venir. ${ }^{46}$

Le monde du roman est le produit de son activité mentale, ce qui explique pourquoi il prise tellement la pensée : “(...) de tous les dons échangés sur terre, c'est encore le don de la pensée qui l'émouvait le plus " (p. 242) ${ }^{47}$; et pourquoi, malade, il ne veut pas prendre de drogues parce qu'elles l'empêcheraient de penser: "Alexandre avait réellement entendu ne pas laisser diminuer son pouvoir de réflexion juste au moment où il lui était plus que jamais nécessaire, 48 .

Pour Elisabeth la pensée n'est pas toujours le même don précieux qu'il est pour Alexandre. Quelquefois elle agit comme si elle était prête à faire n'importe quoi pour étouffer ses pensées. Néanmoins, elle est, comme Alexandre, la créatrice du monde fictif de son roman : “Je me concentre. Je ferme les yeux. J'ai l'air d'évoquer des esprits et pourtant c'est la vie même que je cherche "49. II importe de préciser que Kamouraska n'est pas exactement le récit d'un certain nombre d'événements qui ont abouti à un meurtre et à un procès. Le roman est plutôt ce récit, mais revécu dans la conscience de son protagoniste. L'histoire, telle qu'elle l'a vécue la première fois, s'est passée pour Elisabeth dans un domaine de suspense et d'incertitude. L'histoire revécue n'a cependant plus de lacunes, plus d'ombres, pour elle. Elle a entendu la déposition de tous les témoins : elle a pu reconstruire toute l'histoire en son entier. Dans son étude sur la fin des romans $^{50}$ Alan Friedman a suggéré qu'il suffit de savoir la fin du récit pour posséder une certaine omniscience narrative. Cette omniscience, Elisabeth la possède - non pas pour la séquence d'événements qui se sont produits en réalité, mais pour chaque répétition de cette séquence dans son imagination. Elle est consciente - et fière - de ce pouvoir qu'elle a sur son propre domaine imaginaire :

Jalouse, je veille. Au-delà du temps. Sans tenir compte d'aucune réalité admise. J'ai ce pouvoir. Je suis Mme Rolland et je sais tout. ${ }^{51}$

Elle fait tout marcher. Les événements se déroulent selon le rythme de sa vie intérieure. II suffit qu'elle prononce les noms des villages que doit traverser son amant pour que le trajet se refasse - et si le docteur progresse plus rapidement qu'elle ne le veut, elle n'a qu'à ralentir sa prononciation pour ralentir le progrès du docteur. Voici ce qu'elle se dit en imaginant l'arrivée de son amant à Rivière-Ouelle: «Tenter de faire durer le temps (cinq ou six milles avant Kamouraska). Étirer le plus possible les premières syllabes fermées de ri-vi-, les laisser s'ouvrir en è-re „52. Elle est le Destin qui commande les actions des personnages : 
Mais je suis là, je veux que tu vives et qu'il meure! Je t'ai choisi, toi George Nelson. Je suis la vie et la mort inextricablement liées. Vois comme je suis douce-amere. ${ }^{53}$

Les lois du temps et de l'espace ne s'appliquent plus à son existence, puisqu'il s'agit d'un monde imaginaire dont elle est la maîtresse. Elle est là, «invisible" 54 ; elle sent le froid; elle éprouve la tension et l'angoisse de son amant. Elle devient l'agent qui accomplit l'acte meurtrier :

Que pas une de ses pensées ne m'échappe. Que pas une de ses souffrances ne me soit épargnée. Être deux avec lui. Double et féroce avec lui. Lever le bras avec lui, lorsqu'il le faudra. Tuer mon mari avec lui. ${ }^{55}$

Les deux romans Alexandre Chenevert et Kamouraska présentent, sur le plan humain, des problèmes communs. Quelles solutions proposentils? Kamouraska rejette toute possibilité de solution. J'ai dit que ce roman reste en quelque sorte inachevé parce qu'Elisabeth ne saurait accomplir, en retrouvant son amant, le seul acte qui eût fermé cette expérience. Cet inachèvement existe sur le plan moral aussi, car l'amour qui a donné le seul sens possible à la vie d'Elisabeth, est trahi par son amant. "It is that damned woman that has ruined me "56. Ces mots lui sont communiqués par une jeune connaissance de son amant. Non seulement ils démentent son amour, mais ils le font aussi dans une langue étrangère, celle même de ses juges. La dernière phrase du roman souligne ce sentiment d'aliénation. "Voyez donc comme Madame aime Monsieur! Voyez comme elle pleure", dit la stupide Léontine Melançon. Si Elisabeth pleure, c'est à cause du regret qu'elle éprouve pour sa vie manquée. Elle est à la fin, comme elle l'a été au début, totalement méconnue. II ne lui reste que le rôle ingrat de Mme Rolland.

On pourrait se demander si Alexandre Chenevert n'offre pas une vision plus optimiste du monde. Certes, la dernière phrase du roman paraît contenir un certain optimisme:

II arrive qu'ici et là, dans la ville, quelqu'un dise :

\section{- ... Alexandre Chenevert. ${ }^{57}$}

Cet optimisme semble renforcé par l'esprit d'humanisme et d'espoir qu'acquiert Alexandre pendant sa période à l'hôpital. II convient de savoir, pourtant, si l'attitude de la romancière envers cette dernière phase de la vie de son personnage est fondée ou non sur une ironie pareille à celle qu'affiche Anne Hébert à la fin de Kamouraska. Le bonheur d'Alexandre ne fait pas de doute. L'auteur dit en parlant de son attitude envers le monde: "C'était même curieux à quel point tout cela avait cessé de le tracasser " 58 . Alexandre meurt réconcilié avec un Dieu qu'il s'était mis à détester au nom d'une humanité souffrante : “Alors, il remerciait Dieu qu'il trouvait très bon pour lui, si charitable, si généreux $" 59$. Mais ce nouvel esprit d'optimisme est sujet à caution: "ll est vrai, dit Gabrielle Roy, qu'alors il [Alexandre] prenait une drogue puissante" ${ }^{60}$. Il est fort possible que seul soit réel le désespoir d'Alexandre et que son espoir ne soit que le résultat d'un traite- 
ment médical. Une des rêveries les plus enfantines d'Alexandre concerne son désir d'être atteint d'une maladie incurable afin de devenir une sorte de héros, un martyr gâté par sa famille et ses amis : "ll rêvait du bonheur, dès qu'il serait sur le point de quitter la terre, d'être soigné, compris, regretté peut-être..." ${ }^{61}$. Ces derniers jours d'Alexandre ne seraient-ils donc que la réalisation d'un rêve puéril plutôt qu'un commentaire approfondi sur le sens de la vie?

Ce qui frappe à la fin de cette étude comparative de deux personnages aussi différents qu'Alexandre et Elisabeth, de deux romans aussi différents qu'Alexandre Chenevert et Kamouraska, c'est le grand nombre de points de ressemblance qu'on peut relever entre ces deux ouvrages. Chaque roman présente une expérience commune de la solitude, met en valeur le drame spirituel d'un personnage désespéré cherchant à découvrir sa vraie identité, à trouver une signification à la vie. Aurait-on tort d'aller jusqu'à demander si cette ressemblance existe parce que, malgré leurs différences, les deux romans reflètent un sentiment d'aliénation et une recherche de soi qui sont ceux de la société qu'ils peignent?

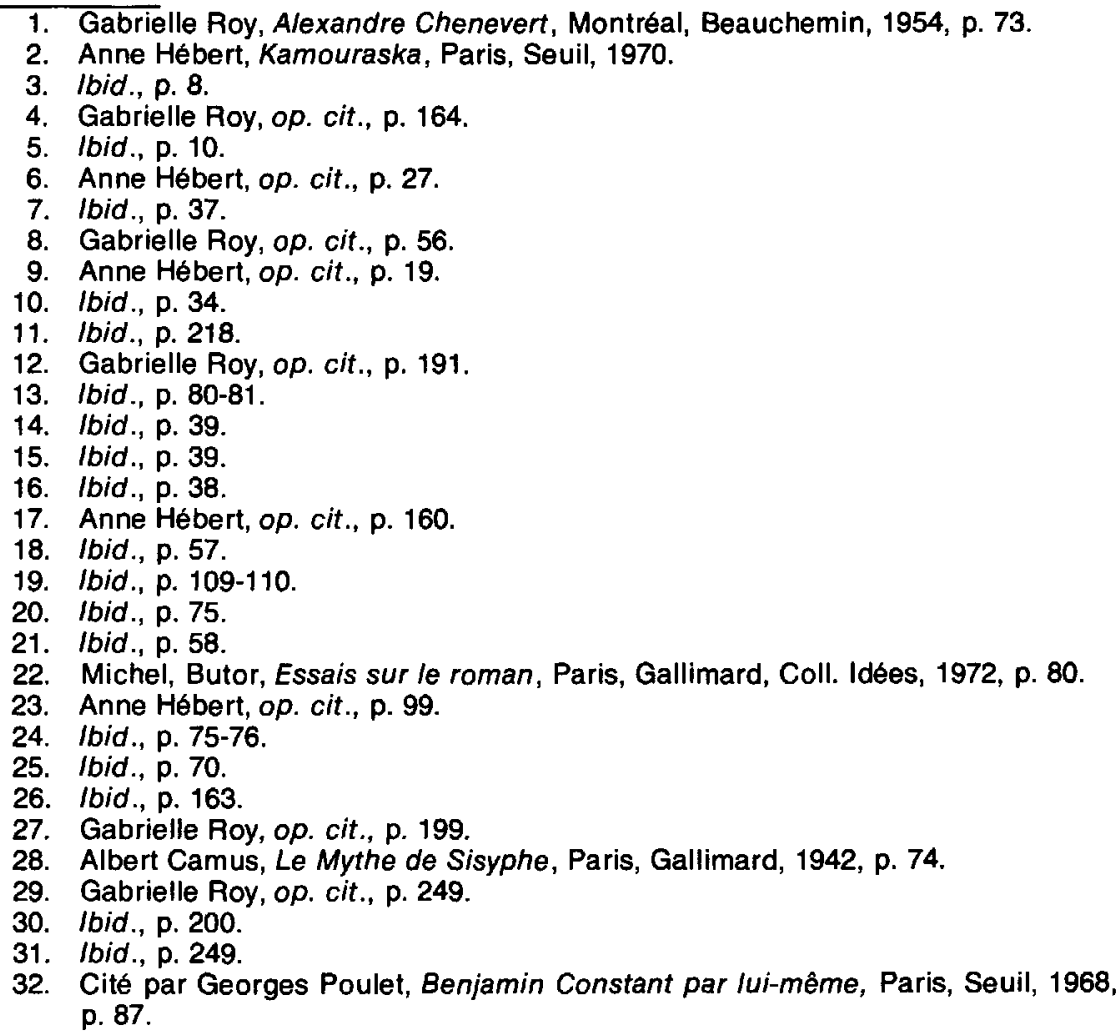


33. Anne Hébert, op. cit., p. 10.

34. Ibid., p. 11.

35. Gabrielle Roy, op. cit., p. 212.

36. Ibid., p. 24.

37. Ibid., p. 99-100.

38. Voir Jean-Paul Sartre, Situations, I, Paris, Gallimard, 1947, p. 33-52.

39. Gabrielle Roy, op. cit., p. 180.

40. Ibid., p. $\mathbf{5 1 .}$

41. Anne Hébert, op. cit., p. 8.

42. Ibid., p. 167.

43. Ibid., p. 208-209.

44. Ibid., p. 158.

45. Gabrielle Roy, op. cit., p. 169.

46. Ibid., p. 146.

47. Ibid., p. 242.

48. Ibid., p. 345.

49. Anne Hébert, op. cit., p. 127.

50. Alan Friedman, The Turn of the Novel, New-York, Oxford University Press, 1967.

51. Anne Hébert, op. cit., p. 126.

52. Ibid., p. 206.

53. Ibid., p. 164.

54. Ibid., p. 215.

55. Ibid., p. 200.

56. Ibid., p. 248.

57. Gabrielle Roy, op. cit., p. 373.

58. Ibid., p. 354.

59. Ibid., p. 365.

60. Ibid., p. 360.

61. Ibid., p. 109. 